\title{
Promoting Healthcare Safety And Quality By Assessing Anticoagulation Education Process
}

\author{
Ali Jenzarli, PhD, University of Tampa, USA \\ Minh-Tri Duong, PharmD, Tampa General Hospital, USA \\ Christy M Thai, PharmD, H. Lee Moffitt Cancer Center \& Research Institute, USA
}

\begin{abstract}
We conducted a baseline study of warfarin patient education process by (1) assessing a healthcare facility's current compliance with the education requirements for anticoagulation therapy, (2) assessing how effectively warfarin education was being provided in terms of patient's knowledge and (3) identifying areas where process improvements were warranted. The facility is an acute care teaching hospital and a Level I Trauma Center with a pharmacist-managed outpatient anticoagulation clinic. We collected data from patients concerning (1) six warfarin knowledge domains (drug-nutrition interactions, drug-drug interactions, monitoring, drug information, dosing and adverse effects), (2) whether or not patients received warfarin education upon discharge and which healthcare professional provided this education (physician, pharmacist, nurse), (3) duration of warfarin therapy, (4) self-rated knowledge of warfarin, and (5) various demographics. Study results indicated the need to implement improvements to the education process to ensure that warfarin education is consistently and routinely provided to all patients prior to being discharged on warfarin with particular attention given to patients sixty years of age and older. Education provided should focus on drug-nutrition and drug-drug interactions, which were found to be areas of highest knowledge deficit. Improvements to the process of providing warfarin education at our hospital may additionally include implementation of a protocol to identify patients requiring education, and a standardized educational program with a pharmacistmanaged warfarin discharge counseling service.
\end{abstract}

Keywords: Anticoagulation Education; National Patient Safety Goals; Oral Anticoagulation Knowledge Test; Warfarin Education; Healthcare Safety and Quality

\section{BACKGROUND}

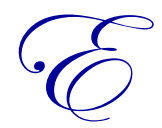

nsuring the safe and appropriate use of anticoagulants remains a challenge despite their frequent use in hospitals. To promote patient safety in the healthcare system, the Joint Commission has taken an active role in improving the implementation and management of anticoagulation therapy. Requirement 03.05.01 of the 2009 National Patient Safety Goals (NPSG) stipulates that hospitals reduce the likelihood of patient harm associated with the use of anticoagulation therapy (Joint Commission, 2009). This requirement has nine elements of performance (EP), two of which address the importance of providing education and evaluating anticoagulation practices as listed below.

- $\quad$ "EP8. The hospital provides education regarding anticoagulation therapy to prescribers, staff, patients, and families."

- $\quad$ "EP9. The hospital evaluates its anticoagulation safety practices, takes appropriate action to improve its practices, and measures the effectiveness of those actions on a regular basis." 
Please notice that the safety goals were revised in 2012 and the reference (Joint Commission, 2009) gives the updated link with EP8 and EP9 above re-numbered EP7 and EP8, respectively.

To address the Joint Commission's performance expectations for education we begin with a baseline assessment of the hospital's current process for providing anticoagulation education. This assessment is an initial step in any quality improvement process, where objective measures are used to identify opportunities for improvement, including areas of non-compliance.

Our assessment focuses on warfarin education because warfarin is one of the primary anticoagulants identified in the rationale for Requirement 03.05.01 (Joint Commission, 2009); and because warfarin is the most widely-prescribed anticoagulant for chronic therapy. In particular, our facility, which is an 877-bed ${ }^{1}$ acute care teaching hospital and a Level I Trauma Center, has a pharmacist-managed outpatient anticoagulation clinic. However in the inpatient setting, oral anticoagulation management is provided by pharmacists through a consult service. Warfarin education is primarily a nursing responsibility and this is typically provided to patients upon discharge.

Due to the complexity of warfarin therapy and associated monitoring requirements, several studies have addressed the relationship between warfarin education/knowledge and its use by patients. In particular, the quality of patient education was shown to be a contributing risk factor in the appropriate and safe use of warfarin (Kagansky et al., 2004). Lack of patient education regarding relevant drug-drug and drug-food interactions, non-adherence to medication therapy, and overall lack of medication knowledge have been shown to contribute to adverse events with warfarin therapy (Taylor et al., 1994). Additionally, a statistically significant positive correlation between patients' knowledge of warfarin and the number of therapeutic International Normalized Ratio (INR) values has been reported (Tang et al., 2003 and Roddie \& Pollock, 1988).

The purpose of this paper is to describe our method for conducting a baseline assessment of the warfarin patient education process. In particular, we conducted a survey study whose objectives were to (1) assess the hospital's current compliance with the education requirements for anticoagulation therapy, (2) assess how effectively warfarin education was being provided in terms of patient's knowledge and (3) identify areas where process improvements are warranted.

\section{METHODS}

Data regarding patient knowledge of oral anticoagulation therapy was collected and analyzed using a validated warfarin knowledge assessment instrument. The instrument was mailed to patients who had received warfarin during their stay at the hospital. In order to identify patients who received warfarin education upon discharge, a report was retrieved using the hospital's pharmacy information system. This report identified patients with active warfarin orders over the course of their hospital stay during any time periods between August 1, 2007 and February 25, 2008. A total of 908 patients were identified as having received warfarin during this period.

Our survey questionnaire was developed based on the oral anticoagulation knowledge test (OAK), a validated instrument developed by Zeolla et al. (2006) to assess patient knowledge of warfarin therapy. The test consists of twenty, individually-validated, multiple-choice questions measuring various knowledge domains. Specifically, these domains were drug-nutrition interactions, drug-drug interactions, monitoring, drug information, dosing and adverse effects with a $7^{\text {th }}$ grade reading level based on the Flesch-Kincaid analysis. Fifteen out of the twenty questions were used in our survey to minimize the time required to complete the survey and promote participation. Five of the questions that we believed measured duplicate knowledge in each domain were omitted from the survey. Patient demographics such as age, race, gender, duration of treatment, and patient's self rating of warfarin knowledge were also assessed. Surveys were mailed to patients' homes and included a self-addressed stamped envelope for return. Test results from this survey represented knowledge acquired through current standards of care, also referred to as the baseline or pre-intervention data. In particular, a knowledge test score was calculated for each respondent as the percentage of correct responses to the fifteen modified-OAK test questions.

\footnotetext{
${ }^{1}$ This was the size of the facility at the time of the study. In 2012 the facility grew to 959 beds.
} 
Our baseline assessment was approved by the Institutional Review Board (IRB) prior to survey distribution. Depending on results from this assessment, process improvements will be made in order to meet the required education expectations.

In the event that interventions would have to be made to improve patient anticoagulation education, a sample size was pre-determined to allow for pre- and post-intervention assessments. Using a two-sided, paired t-test with a 0.05 level of significance, a standard deviation of $23 \%$ and a power of $80 \%$, a sample size of 24 patients was needed to detect a mean score difference of $15 \%$ between pre- and post-aggregate test scores. Anticipating a mailout response rate of $15 \%$, a total of 160 surveys were randomly distributed with the goal of obtaining a minimum of 24 returned surveys. Results obtained from the surveys were analyzed using descriptive and inferential statistics.

\section{RESULTS}

One-hundred-sixty surveys were mailed to patients, of which thirty-five (35) usable surveys were returned to the pharmacy for a better-than-expected response rate of 21.9\%. Patient demographics are shown in Table 1 . The mean age of our patients was 65.3 years and the median age was 65 years, where $31.4 \%$ were less than 60 years of age. The percentage of female patients was $8.6 \%$ higher than that of male patients. The vast majority of patients were Caucasian (91.4\%). Table 1 also shows the percentage distribution of the duration of warfarin therapy, where the majority of patients $(57.1 \%)$ were on warfarin between 6 months and 5 years. Almost $29 \%$ of patients were on warfarin less than 6 months, and less than $15 \%$ of patients were on warfarin more than 5 years.

Table 1: Patient Demographics

\begin{tabular}{|l|c|}
\hline \multicolumn{2}{|c|}{ Mean Age (years) } \\
\hline All Patients & Median Age (years) \\
\hline & 65 \\
\hline All Patients & Patients (\%) \\
\hline Age Range & 31.4 \\
\hline$<60$ & 68.6 \\
\hline$>=60$ & Gender (\%) \\
\hline Female & 54.3 \\
\hline Male & 45.7 \\
\hline Caucasian & Ethnicity (\%) \\
\hline African American & 91.4 \\
\hline Hispanic/Latino & 2.9 \\
\hline Other (not specified) & 2.9 \\
\hline Duration of warfarin therapy & 2.9 \\
\hline 6 months & Patients (\%) \\
\hline m months - 1 year & 28.6 \\
\hline 1 year- 5 years & 25.7 \\
\hline$>$ 5 years & 31.4 \\
\hline
\end{tabular}


Considering all 35 usable surveys the aggregate mean score on the modified-OAK test was $72.4 \%$. Twentytwo patients $(62.9 \%)$ reported receiving no warfarin education and their mean test score was $70.6 \%$. Only thirteen patients $(37.1 \%)$ reported receiving warfarin education and their mean test score was $75.4 \%$. At the $\alpha=.05$ level of significance, the difference between the mean test score for patients receiving warfarin education and the mean test score for patients receiving no education is not statistically significant with a p-value of .30 (Table 2).

Considering the 13 patients who received education, pharmacists provided the education to six or $46.2 \%$ of these patients, nurses provided the education to five or $38.4 \%$, and physicians provided the education to two or $15.4 \%$. Patients who reported receiving warfarin education from a pharmacist had a mean test score of $87.8 \%$, statistically significantly greater than the mean test score of $64.8 \%(\alpha=.05$, p-value $=.006)$ achieved by those who reported receiving warfarin education from other healthcare professionals (Table 2).

Table 2: Warfarin Education and Associated Mean Test Scores

\begin{tabular}{|l|c|c|}
\hline \multicolumn{1}{|c|}{ Warfarin education } & Number of Patients (\% out of 35) & Mean test score (\%) \\
\hline No & $22(62.9 \%)$ & 70.6 \\
\hline Yes & $13(37.1 \%)$ & 75.4 \\
\hline By Physician & 2 & 63.3 \\
\hline By Pharmacist & 6 & 87.8 \\
\hline By Nurse & 5 & 65.3 \\
\hline By Other than Pharmacist & 7 & 64.8 \\
\hline
\end{tabular}

Mean test scores were compared for different groups of patients based on the duration of their warfarin therapy. Patients who had been on warfarin for more than one year had a mean test score of $80 \%$, statistically significantly greater than the mean test score of $66 \%$ for those patients on warfarin for less than one year $(\alpha=.055$, $\mathrm{p}$-value $=.053)$. More specifically, patients on warfarin for less than six months had a mean score of $63.3 \%$ while patients on warfarin for six to twelve months had a mean score of $68.9 \%$. Patients on warfarin for 1-5 years had a mean score of $83.6 \%$ and those on warfarin for more than five years had a mean score of $72 \%$ (Table 3 ).

Table 3: Duration of Warfarin Therapy and Associated Mean Test Scores

\begin{tabular}{|c|c|c|}
\hline Warfarin education & Number of Patients & Mean test score (\%) \\
\hline$<6$ months & 10 & 63.3 \\
\hline 6 months - 1 year & 9 & 68.9 \\
\hline 1 year- 5 years & 11 & 83.6 \\
\hline$>5$ years & 5 & 72 \\
\hline
\end{tabular}

Considering the sixteen patients who had been on warfarin for more than a year, the mean test score of the six patients who reported receiving education was $80 \%$, equal to the mean test score of the ten patients who reported receiving no education.

Mean test scores were compared for different age groups of patients (Table 4). Patients less than 60 years of age had a mean test score of $85.5 \%$, statistically significantly greater than the mean test score of $66.4 \%$ achieved by patients 60 years of age and older $(\alpha=.05$, p-value $=.02)$.

Table 4: Age Range and Associated Mean Test Scores

\begin{tabular}{|c|c|}
\hline Age Range & Mean test score (\%) \\
\hline$<60$ & $85.5 \%$ \\
\hline$>=60$ & $66.4 \%$ \\
\hline
\end{tabular}

Patients had the option of rating their knowledge of warfarin as excellent, very good, good, fair or poor. Sixty percent of patients rated their warfarin knowledge as good or better. Twenty-six percent rated their warfarin knowledge as fair, and $14.3 \%$ rated their knowledge as poor. A positive relationship was seen between self-rated warfarin knowledge and associated mean test scores. The one patient who rated his/her knowledge as excellent had a test score of $86.7 \%$. Patients who rated their knowledge as very good and good had a mean test score of $83.7 \%$ and $80 \%$, respectively. However, patients with fair or poor self-rated knowledge had a mean test score less than $58.1 \%$. 
Finally, the modified-OAK test questions were stratified based on warfarin knowledge domains. Each knowledge domain had at least two distinct questions, each of which measured a distinct dimension of that knowledge domain. The percentage of correct responses to all of the dimensional questions of a particular knowledge domain was calculated and used as an indicator of patients' level of knowledge of that domain (Table 5). The highest levels of knowledge were found in the domains of dosing and adverse effects, where only $65.7 \%$ of patients correctly answered all dimensional questions of each of these domains. The lowest levels of knowledge were found in the domain of drug-nutrition interactions, where a mere $17.1 \%$ of patients correctly answered all dimensional questions of this domain.

Table 5: Percentage of Correct Responses to All Questions on Each Knowledge Domain

\begin{tabular}{|l|c|}
\hline \multicolumn{1}{|c|}{ Warfarin knowledge domain } & \% of correct responses out of 35 \\
\hline Drug-nutrition interactions & 17.1 \\
\hline Drug-drug interactions & 40 \\
\hline Monitoring & 54.3 \\
\hline Drug information & 60 \\
\hline Dosing & 65.7 \\
\hline Adverse effects & 65.7 \\
\hline
\end{tabular}
domain.

Table 6 shows the percentage of correct responses to each dimensional question in each knowledge

Table 6: Percentage of Correct Responses to Each Question on Each Knowledge Domain

\begin{tabular}{|l|c|}
\hline \multicolumn{1}{|c|}{ Warfarin knowledge domain (Question \#) } & \% of correct responses out of 35 \\
\hline Drug-nutrition interactions (Q3, Q5, Q15) & $(57.1,71.4,42.9)$ \\
\hline Drug-drug interactions (Q6, Q12, Q14) & $(48.6,85.7,82.9)$ \\
\hline Monitoring (Q4, Q7, Q11) & $(60,3,65.7,88.6)$ \\
\hline Drug information (Q1, Q13) & $(65.7,88.6)$ \\
\hline Dosing (Q2, Q8) & $(91.4,68.6)$ \\
\hline Adverse effects (Q9, Q10) & \\
\hline
\end{tabular}

\section{DISCUSSION}

Based on results from the returned surveys, thirteen of the thirty-five patients $(37.1 \%)$ surveyed reported receiving warfarin education. This means that a $95 \%$ confidence interval estimate of the population percentage of patients receiving warfarin education upon discharge was between $21 \%$ and $53 \%$. This confidence interval estimate fell short of the Joint Commission's mandated compliance percentage of 100\%. Furthermore, results showed that even when warfarin education was provided, it was provided by various health care professionals; pharmacists provided education to six, nurses to five and physicians to two of the thirteen patients.

We consider a score of $90 \%$ on the modified-OAK test to be a standard score signifying adequate knowledge of warfarin therapy. The aggregate mean test score of $72.4 \%$ showed that patients' knowledge of warfarin therapy could be improved. In particular, the patient population mean test score was between $63.6 \%$ and $81.1 \%$ with $95 \%$ confidence, an interval estimate that did not contain the standard score of $90 \%$. Furthermore, sample results showed that there was no statistically significant difference between population mean test scores of those patients who received education (75.4\%) and those who did not (70.6\%) at the .05 level of significance. Again, the $95 \%$ confidence interval estimate for each population mean test score did not contain the standard score of $90 \%$.

Sample results showed that population mean test score of patients who reported receiving warfarin education from a pharmacist was statistically significantly greater than population mean test score of patients who reported receiving warfarin education from other healthcare professionals at the .05 level of significance. Moreover, sample statistics for those patients who received education from a pharmacist yielded a 95\% confidence interval estimate for the population mean test score $(82.5 \%, 93.04 \%)$ that contained the standard score of $90 \%$. This finding was encouraging even though it was based on a small sample of only six patients (Table 2). 
Mean test scores appeared to increase as patients were maintained on warfarin therapy. This was anticipated since we would expect a positive relationship between patient knowledge of medications and their duration of therapy. Patients on warfarin for an extended period of time would be expected to have had more opportunities for patient education. However, this positive relationship dissipated after 5 years of warfarin therapy. Patients who had been on warfarin for one to five years scored higher, but not statistically significantly so, on average than those on warfarin for more than five years, whereas patients on warfarin for more than one year scored statistically significantly higher than patients who had been on warfarin for less than one year at the .055 level of significance. This may have been attributed to the lack of warfarin education that was provided to patients who had been on warfarin for more than 5 years, since none of these patients received education upon discharge. Lack of follow-up education for these patients may have been due to the incorrect assumption that patients on warfarin for a prolonged period of time were already knowledgeable about their warfarin therapy.

Another encouraging finding was that sample statistics for those patients who had been on warfarin for 1-5 years yielded a 95\% confidence interval for the population mean test score $(74.8 \%, 92.5 \%)$ that contained the standard score of $90 \%$. However, this encouraging finding was tempered by the fact that for patients who had been on warfarin for more than a year, there was no difference, statistically significant or otherwise, between mean test scores of those patients who received education and those who did not.

Mean test scores appeared to decrease as patients got older, where patients less than 60 years of age had a population mean test score that was statistically significantly greater than that of patients 60 years of age and older. For those patients less than 60 years of age sample results yielded a $95 \%$ confidence interval for the population mean test score $(77.3 \%, 93.7 \%)$ that contained the standard score of $90 \%$. This represents yet another encouraging finding that also informs future interventions to pay particular attention to patients 60 years of age and older.

Sample results for warfarin knowledge domains indicated inadequate levels of knowledge across all six domains (Table 5), where the lowest levels were found in the domains of drug-nutrition and drug-drug interactions. None of the sample percentages of correct responses in Table 5 yielded a 95\% confidence interval estimate for the population mean percentage of correct responses that contained the standard score of $90 \%$. However, Table 6 shows that at least one dimensional question in each domain, except for the drug-nutrition interaction domain, had a sample percentage of correct responses that yielded a 95\% confidence interval estimate that contained the standard score of 90\%. These dimensional questions are Q12 on drug-vitamins interactions, Q14 on drug-pain reliever interactions, Q11 on when to contact other healthcare providers, Q13 on usage of warfarin, Q8 on what to do when missing a dose, and Q9 on when to monitor for signs of bleeding (Appendix 1).

Our institution's process for providing warfarin education needs improvement to meet current requirements of the Joint Commission. Education process improvements should focus on implementing a process to ensure that warfarin education is routinely and consistently provided to all patients receiving warfarin at the hospital. Education improvements should also focus on all knowledge domains with particular emphasis on drug-nutrition interactions, where none of the dimensional questions met our standard, and only on those dimensional questions in the other knowledge domains that did not meet our standard.

A positive effect was seen when patients reported receiving warfarin education from a pharmacist. The potential for implementing pharmacist-managed warfarin education will be explored since preliminary results indicate an improvement in warfarin education with pharmacist interventions.

There are several limitations to this baseline assessment. The majority of patients in our sample were Caucasian, which may limit the ability to generalize the study results to all patients on warfarin at the hospital since this ethnic composition is not representative of our patient population. Non-response and voluntary response bias may also affect the results of the study. Patients' tendency to complete the surveys may have been influenced by their perceived knowledge of warfarin. Patients who are unwilling or unable to participate in the survey may contribute to non-response bias. Voluntary response bias may be an issue as patients are self-selected volunteers. Patients more knowledgeable about warfarin may have been more likely to complete and return the surveys, falsely inflating the mean test score. Patients' previous warfarin knowledge was also not assessed and it is possible that patients in our study were already well educated on warfarin. The mean test score may have also been elevated by the potential for patients to have looked up answers to the various questions, since surveys were not completed 
under supervision. Finally, some of our findings concerning education provided by healthcare professionals may have been tempered by the small size (13) of our sample of patients who reported receiving education.

\section{CONCLUSION}

Based on results from this study, education process improvements will be implemented to ensure that warfarin education is consistently and routinely provided to all patients prior to being discharged on warfarin. Education provided will focus on drug-nutrition and drug-drug interactions, which were found to be areas of highest knowledge deficit. Improvements to the process of providing warfarin education at our hospital may additionally include the implementation of a protocol to identify patients requiring education and a standardized educational program and/or a pharmacist-managed warfarin discharge counseling service.

Considering our assessment objectives listed in the background section, we believe (1) that the hospital's process for anticoagulation therapy education needs improvement to comply with the Joint Commission's performance expectations, (2) that education, in terms of patient's knowledge, needs improvement, and (3) that we were able to identify areas where process improvements are warranted.

\section{IMPLICATIONS}

Post-assessments will be conducted using the same survey instrument and methods to evaluate process improvements implemented. This study exemplifies the importance of conducting an analysis of current anticoagulation education practices and identifying potential areas of non-compliance. We found that utilizing a modified version of the oral anticoagulation knowledge test was extremely helpful in assessing our patients' knowledge of warfarin. This study emphasizes the importance of completing a state of analysis before implementing change, which will help identify opportunities for improvement and help direct the institution toward meeting goal compliance.

\section{AUTHOR INFORMATION}

Ali Jenzarli, PhD is currently Associate Professor of Information and Technology Management at Sykes College of Business, University of Tampa, Tampa, Florida, USA. Dr. Jenzarli has conducted more than 50 local, national and international consulting projects in operations, software and business valuations, data analysis, forecasting, quality and process improvement, and program accreditation. He has more than 100 intellectual contributions in basic and applied research including manuscripts published in peer-reviewed journals and conference proceedings, peerreviewed presentations, new course and workshop developments and seven grants. Topics include project, operations, quality and risk management, business intelligence, forecasting, uncertain reasoning, artificial intelligence, health environments design and clinical data analysis. He holds a Ph.D. in management science with concentrations in applied statistics and artificial intelligence from the University of Kansas, and a B.S. in electrical and computer engineering from the University of Louisiana-Lafayette. He has received 19 honors and awards for outstanding teaching, service and academic achievements. He has held more than eight leadership positions in industry, university and community organizations and is a member of six professional societies and institutes. E-mail: ajenzarli@ut.edu (Corresponding author)

Minh-Tri Duong, PharmD is currently the Director of Residency Programs and Education Coordinator at Tampa General Hospital. Her practice includes managing the department's educational programs and expanding and optimizing pharmacy clinical services. Dr. Duong received her Doctor of Pharmacy Degree from the University of Florida, Gainesville, Florida and went on to complete an ASHP accredited specialty residency in ambulatory care at Florida Hospital in Orlando, FL. Dr. Duong has worked as a Clinical Pharmacist in outpatient and inpatient settings and was Assistant Professor of pharmacy practice at Florida A\&M University. Work experiences include primary care Clinical Pharmacist with focus on anticoagulation, hyperlipidemia, diabetes, hypertension, and pharmacokinetics services; Clinical Liaison between pharmaceutical industry and national opinion leaders to develop professional educational programs for pharmacy and physician groups; Clinical Pharmacist and Certified Speaker for continuing education programs. Research interest includes clinical outcome studies and service improvement initiatives. She has presented at professional state meetings and has been accepted for peer review publication. E-mail: mduong@tgh.org 
Christy Thai, PharmD is currently a clinical pharmacist with the Internal \& Hospital Medicine service at Moffitt Cancer Center. She earned her Doctor of Pharmacy Degree from the University of Florida (2007) and completed a Pharmacy Practice residency at Tampa General Hospital (2008). Her pharmacy experience includes hospital inpatient services and outpatient anticoagulation management. Dr. Thai's research interest includes anticoagulation therapy and increasing education and awareness among pharmacists, pharmacy students, and medical residents regarding the appropriate use of medications in oncology patients. She is actively involved in establishing and improving anticoagulation protocols at her current practice site and serves as a preceptor for both pharmacy residents and students. Email: christy.thai@moffitt.org

\section{REFERENCES}

1. Joint Commission (2009). 2009 National Patient Safety Goals [Accessed September 5, 2009]. Available from: http://www.jointcommission.org/NR/rdonlyres/F71BC4E9-FEB6-495C-99D8DB9F0850E75B/0/09_NPSG_General_Presentation.ppt, and updated at the following link. http://www.jointcommission.org/assets/1/6/NPSG_Chapter_Jan2012_CAH.pdf, pages 3-4.

2. Kagansky N, Knobler H, Rimon E, et al. (2004). Safety of Anticoagulation Therapy in Well-informed Patients. Arch Intern Med, 164:2044-50.

3. Roddie AMS, Pollock A (1988). Therapeutic Control of Anticoagulation: How Important is Patient Education? Clin Lab Haematol, 10:109-12.

4. Tang EOYL, Lai CSM, Lee KKC, et al. (2003). Relationship Between Patients' Warfarin Knowledge and Anticoagulation Control. Ann Pharmacother, 37:34-9.

5. Taylor FC, Ramsay ME, Tan G, et al. (1994). Evaluation of patients' Knowledge about Anticoagulant Treatment. Quality in Health Care, 3(2) 79-85.

6. Zeolla M, Brodeur M, Dominelli A, et al. (2006). Development and Validation of an Instrument to Determine Patient Knowledge: The Oral Anticoagulation Knowledge Test. Ann Pharmacother, 40:633-8. 


\section{APPENDIX 1}

\section{Directions for completing the survey:}

- $\quad$ Answer each question. DO NOT leave questions unanswered.

- Please check the box or fill in the blank that most closely represents your response.

1. How old are you? ___ years

2. What is your gender?

$\square$ Male

Female

3. What is your race? (Choose one)

$\square$ African-American

$\square$ Hispanic/Latino

$\square$ White/Caucasian

$\square$ Asian

$\square$ Other (please specify)

4. How long have you been on warfarin (Coumadin $®)$ ? (Choose one)

$\square<6$ months

6 months -1 year

$\square 1$ year -5 years

$\square>5$ years

5. How would you rate your knowledge of warfarin (Coumadin $\left.{ }^{\circledR}\right)$ ? (Choose one)

$\square$ Poor

$\square$ Fair

$\square$ Good

$\square$ Very good

Excellent

6. Did you receive education about warfarin (Coumadin $\left.{ }^{\circledR}\right)$ during your hospital stay?

$\square$ Yes

No

7. If you answered "YES" to question \#6, please tell us who provided the education about warfarin? (Choose one)

$\square$ Nurse

$\square$ Pharmacist

$\square$ Physician

Other (please specify)

8. Did a pharmacist meet with you during your stay in the hospital?

$\square$ Yes

$\square$ No

\section{****Please only check ONE box per question***}

1. Different strengths of warfarin (Coumadin $®)$ will differ in:

$\square$ color

$\square$ shape

$\square$ size

$\square$ weight

2. Missing one dose of warfarin (Coumadin $®)$ can:

cause your PT/INR ("protime") to be above the goal range

$\square$ increase your risk of bleeding

$\square$ cause your PT/INR ("protime") to be below the goal range

$\square$ decrease your risk of having a clot 
3. Occasionally eating a large amount of leafy green vegetables while taking warfarin (Coumadin $®)$ can: $\square$ increase your risk of bleeding $\square$ increase your risk of having a blood clot $\square$ cause stomach upset and vomiting

reduce your risk of having a blood clot

4. The PT/INR ("Protime") test is:

$\square$ a blood test used to monitor therapy with warfarin (Coumadin®)

$\square$ a blood test that is used to monitor drug interactions with warfarin (Coumadin)

$\square$ a blood test that checks the amount of vitamin $\mathrm{K}$ in your diet

$\square$ a blood test that determines if you need to be on warfarin (Coumadin $\left.{ }^{\circledR}\right)$

5. Drinking alcohol while taking warfarin (Coumadin $\left.{ }^{\circledR}\right)$ :

$\square$ is safe as long as you separate your dose of warfarin (Coumadin $\left.{ }^{\circledR}\right)$ and consumption of alcohol

$\square$ may affect your PT/INR ("protime")

$\square$ does not affect your PT/INR ("protime")

$\square$ is safe as long as you are on a low dose

6. Which of the following over-the-counter products is most likely to interact with warfarin (Coumadin $\left.{ }^{\circledR}\right)$ ?

$\square$ nicotine replacement therapies

$\square$ herbal/dietary supplements

allergy medications

calcium supplements

7. A patient with a PT/INR ("protime") value above the "goal range":

$\square$ is at increased risk of having a clot

$\square$ is more likely to have drowsiness and fatigue from warfarin (Coumadin ${ }^{\circledR}$ )

$\square$ is at increased risk of bleeding

is less likely to experience side effects from warfarin (Coumadin $\left.{ }^{\circledR}\right)$

8. The best thing to do if you miss a dose of warfarin (Coumadin $\left.{ }^{\circledR}\right)$ is to?

$\square$ double up the next day

$\square$ take the next scheduled dose and tell your healthcare provider

$\square$ call your healthcare provider immediately

discontinue warfarin (Coumadin $®)$ altogether

9. It is important for a patient on warfarin (Coumadin $\left.{ }^{\circledR}\right)$ to monitor for signs of bleeding:

$\square$ only when their PT/INR ("protime") is above the goal range

$\square$ at all times

$\square$ only when their PT/INR ("protime") is below the goal range

$\square$ only when you miss a dose

10. A person on warfarin (Coumadin $®)$ should seek immediate medical attention:

if they skip more than two doses of warfarin (Coumadin $\AA$ ) in a row

$\square$ if they notice blood in their stool when going to the bathroom

$\square$ if they experience a minor nosebleed

if they develop bruises on their arms or legs

11. A patient on warfarin (Coumadin $\left.{ }^{\circledR}\right)$ therapy should contact the physician or healthcare provider when: another physician adds a new medication

another physician stops a current medication

$\square$ another physician changes a dose of a current medication

all of the above

12. Which of the following vitamins interacts with warfarin (Coumadin $®)$ ?

$\square$ vitamin B12

vitamin A

$\square$ vitamin B6

vitamin $\mathrm{K}$ 
13. Warfarin (Coumadin $\left.{ }^{\circledR}\right)$ may be used to:

$\square$ treat people that already have a blood clot

treat people that have high blood sugar levels

$\square$ treat people with high blood pressure

treat people with severe wounds

14. Taking a medication containing aspirin or other over-the-counter pain reliever such as ibuprofen (Motrin ${ }^{\circledR}$, Advil@) while on warfarin (Coumadin $®)$ will:

reduce the effectiveness of the warfarin (Coumadin $\left.{ }^{\circledR}\right)$

increase your risk of bleeding from the warfarin (Coumadin $®$ )

$\square$ cause a blood clot to form

require you to increase your dose of warfarin (Coumadin $\left.{ }^{\circledR}\right)$

15. When it comes to diet, people taking warfarin (Coumadin $®)$ should:

never eat foods that contain large amounts of vitamin $\mathrm{K}$

keep a diary of all of the foods they eat

$\square$ be consistent and eat a diet that includes all types of foods

increase the amount of vegetables they eat

This is the end of the survey.

Thank you for taking the time to answer ALL questions.

***Please remember to return this survey by mailing it in using the envelope provided. No postage is necessary. ${ }^{* * *}$ 
NOTES 